\title{
Multi-slice spiral CT evaluation of chronic radiation colitis and rectitis
}

\author{
JING XIAO and QING-DONG LI \\ Key Laboratory for Biorheological Science and Technology of the Ministry of Education (Chongqing University), \\ Chongqing University Cancer Hospital, Chongqing 400044, P.R. China
}

Received September 5, 2019; Accepted May 22, 2020

DOI: $10.3892 /$ etm.2020.9069

\begin{abstract}
The aim of the present study was to retrospectively analyse the multi-slice spiral CT (MSCT) findings of radiation colitis and rectitis (RC\&R). A total of 23 cases of RC\&R detected by helical CT were included. The CT findings and clinical and endoscopy data of the patients were reviewed. The primary tumours included cancers of the cervix $(n=17)$, rectum $(n=4)$, ovaries $(n=1)$ and bladder $(n=1)$. The total dose of radiation per patient was 46-60 Gy (mean, 49.7 Gy) delivered over 5 weeks. The CT manifestations included different degrees of increased thickness of the intestinal wall $(n=20,87.0 \%)$, with a maximum thickness of $16.6 \mathrm{~mm}$. On enhanced CT, the target sign was observed $(n=16,69.9 \%)$, with an obviously enhanced mucosa and/or serosa and the following changes observed: Oedema and increased density of the mesentery $(n=15,65.2 \%)$; increased density of the subcutaneous fat, and blurred and oedematous pelvic wall muscles $(n=4,17.4 \%)$, with the obturator internus and levator ani muscles being most commonly affected; narrowed intestinal lumen $(n=3$, $13.0 \%)$; and a small amount of ascitic fluid $(n=2,8.7 \%)$ located in the paracolic sulci and bladder or Douglas pouch. The 23 patients underwent colonoscopy and were diagnosed with RC\&R. The major manifestations included telangiectasia and mucosal hyperaemia $(n=21,91.3 \%)$. MSCT of chronic RC\&R (CRC\&R) was associated with certain characteristic findings, which, combined with a medical history of radiotherapy and the clinical manifestations, may prove to be of value in the diagnosis of CRC\&R.
\end{abstract}

\section{Introduction}

Radiation therapy is a major component of the armamentarium for the treatment of various malignancies. Regardless

Correspondence to: Professor Qing-Dong Li, Key Laboratory for Biorheological Science and Technology of the Ministry of Education (Chongqing University), Chongqing University Cancer Hospital, 181 Hanyu Road, Chongqing 400044, P.R. China

E-mail: 1515607901@qq.com

Key words: radiotherapy, radiation enteritis, imaging, CT of the tumour cell type, radiation may have a central, adjuvant or neoadjuvant role. In several gynaecological, urological and rectal cancers, radiation treatment is limited to the pelvis, where a maximum dose may be delivered to the target tissue while minimizing inadvertent irradiation of other organs. Unfortunately, the radiation fields required to treat these pelvic and, occasionally, intra-abdominal or retroperitoneal tumours, frequently include the small bowel and colon (1).

With the increased incidence of malignant tumours, the higher long-term survival rate of cancer patients and the increased popularity of radiotherapy for the treatment of pelvic malignant tumours, the incidence of radiation enteritis (RE), a common post-radiotherapy complication, has been continuously increasing $(2,3)$. Based on its clinical onset, RE may be generally classified as acute or chronic (4). With regard to acute RE (ARE), most cases are self-healing and require no special treatment. However, chronic RE (CRE) is associated with more severe latent and progressive manifestations, increased pain and reduced quality of life, requiring surgical treatment in $>30 \%$ of patients (5). Therefore, the diagnosis of CRE has been attracting increasing clinical attention. As the most commonly involved organs are the colon and rectum, pelvic RE is also referred to as radiation colitis and rectitis (RC\&R). Only few studies have investigated the diagnosis of RC\&R (6). Therefore, in the present study, clinical, endoscopic and imaging data of 23 patients with CRC\&R were retrospectively analysed to explore the diagnostic value of multi-slice spiral CT (MSCT).

\section{Materials and methods}

Patient selection and eligibility. The present study was a retrospective, observational study. From a database of the internal medical records system, a list of 151 patients with endoscopically proven RC\&R who underwent pelvic CT examination at Chongqing University Cancer Hospital (Chongqing, China) over a 6-year period (from May 2013 to May 2019) was obtained. Among those, 23 patients were finally selected after reviewing their radiological as well as diagnostic pathological and medical records. The diagnosis of RC\&R in these selected patients had been based on a history of radiotherapy for malignant pelvic tumours, as well as pelvic CT prior to radiotherapy displaying no abnormal changes in the pelvic intestinal tract and follow-up examination after 


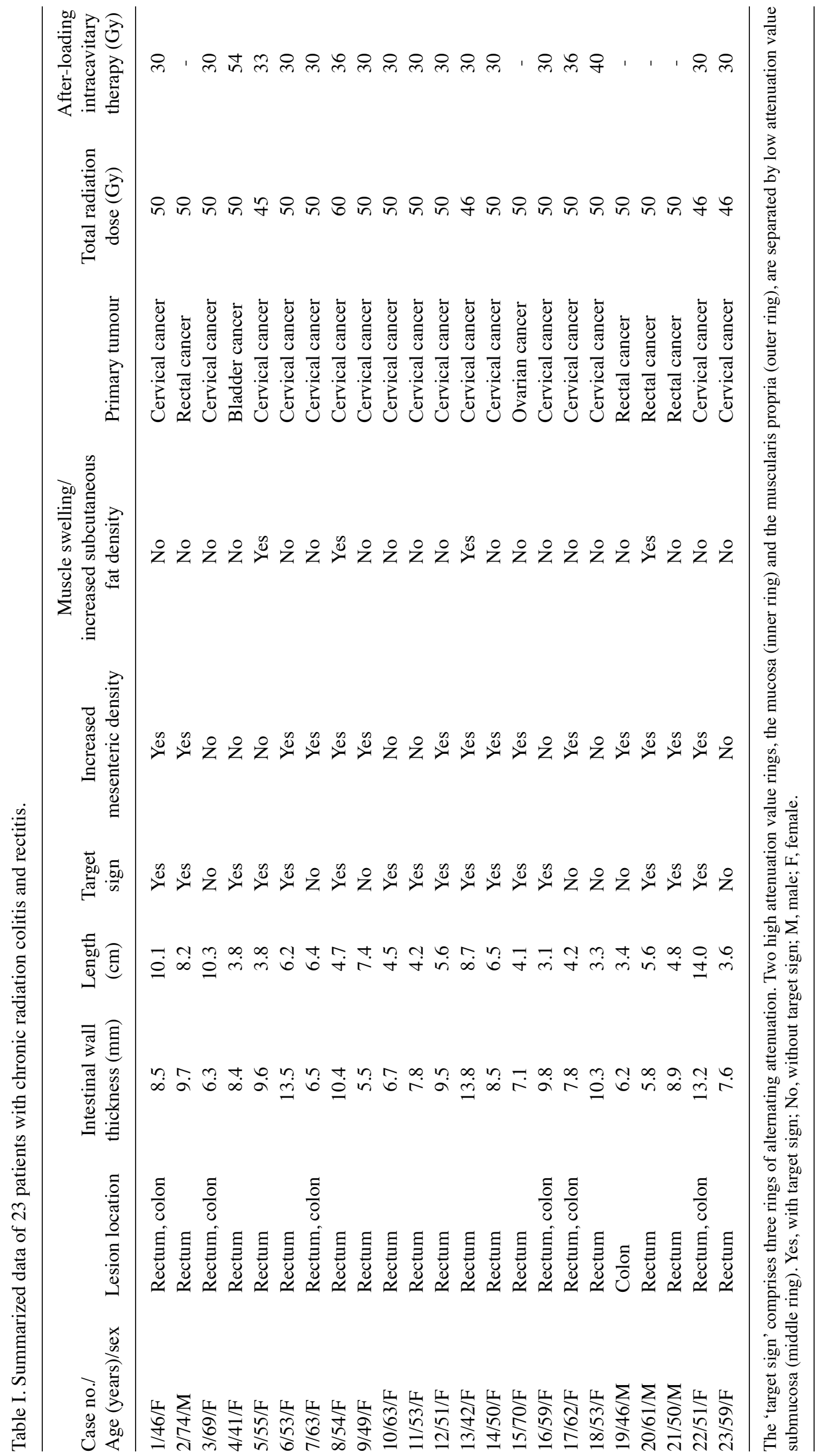


radiotherapy included an enhanced pelvic CT scan. There was a latent period ( $>3$ months) with typical clinical manifestations of CRE after radiotherapy (7). All diagnoses were confirmed endoscopically. Patients without complete CT and endoscopic data were excluded as were patients diagnosed with primary and recurrent intestinal tumours, as well as primary inflammatory and ischemic intestinal diseases. The patient population comprised 4 males and 19 females, ranging in age from 41-74 years (mean, 53 years).

CT technique. CT was performed using a Philips 64-slice spiral CT machine (Philips Medical Systems, Inc.). The scanning range was from the inferior margin of the pubic symphysis to the superior umbilicus or lower diaphragm. All patients were examined by plain and enhanced CT. For the latter, 80-100 $\mathrm{ml}$ of a non-ionic iodinated contrast agent was injected via the elbow vein with a high-pressure injector at the flow rate of 2.5-3.0 ml/sec. Scanning was initiated 50-60 sec after the injection. The reformation slice thickness and slice gap of the transverse section and sagittal section were 3 and $5 \mathrm{~mm}$, respectively.

Statistical analysis. The CT findings were retrospectively reviewed by the consensus of two experienced radiologists. The CT scans were evaluated with an emphasis on the following: Site of involvement, thickness of the intestinal wall, length of the lesion, presence or absence of a layering pattern in the involved segment of the bowel wall [known as the target sign, the target sign comprises three rings of alternating attenuation. Two high attenuation value rings, the mucosa (inner ring) and the muscularis propria (outer ring) are separated by the low attenuation value submucosa (middle ring)], resolution of the pericolic fat tissue, density of subcutaneous fat and presence of pelvic abscesses or intestinal fistulas. The clinical, colonoscopic and pathological findings were evaluated simultaneously. All analyses were conducted using SPSS 16.0 (SPSS Inc.). In order to determine whether the thickness of the intestinal wall was associated with the presence of the target sign, the cases were divided into $\leq 6.5$ and $>6.5-\mathrm{mm}$ groups, and the $\chi^{2}$ test or Fisher's exact probability method was used. $\mathrm{P}<0.05$ was considered to indicate a statistically significant difference.

\section{Results}

Patient characteristics. The characteristics of the 23 patients are summarized in Tables I and SI.

The primary tumours included cancer of the cervix $(n=17)$, rectum $(n=4)$, ovaries $(n=1)$ and bladder $(n=1)$. The total dose of radiation per patient was 46-60 Gy (mean, 49.7 Gy) delivered over 5 weeks. Among the patients, those with cervical cancer $(n=17)$ and bladder cancer $(n=1)$ were treated using after-loading intracavitary therapy with a total dose of 30-54 Gy (mean, 32.7 Gy). The major clinical symptoms included increased frequency of defecation $(n=16)$, abdominal pain $(n=12)$ and constipation and tenesmus $(n=5)$, while there was a lack of obvious symptoms in 3 cases (Table II). Endoscopy and CT examination were performed from 5 to 29 months after radiotherapy. Of the 23 patients, 22 exhibited involvement of the rectum $(96.7 \%), 7$ of the sigmoid colon
Table II. Major clinical symptoms.

\begin{tabular}{lc}
\hline Clinical symptoms & $\mathrm{N}(\%)$ \\
\hline Increased frequency of defecation & $16(69.6)$ \\
Abdominal pain & $12(52.2)$ \\
Constipation and tenesmus & $5(21.7)$
\end{tabular}

(30.5\%) and 6 exhibited both (26.1\%). The length of the affected intestinal segment varied from 3.1 to $14.0 \mathrm{~cm}$.

CT manifestations. The patients exhibited different degrees of increased thickness of the intestinal wall $(n=20,87.0 \%)$, with a maximum of $16.6 \mathrm{~mm}$, as exemplified in the representative case in Fig. 1. The intestinal wall was indicated to be thickened, with smooth contours and no obvious protrusions or masses. The target sign $(n=16,69.9 \%)$ on enhanced CT was observed as a significant enhancement of the mucosal and/or serosal layers, presenting a stratified change. The target sign was more commonly observed when the thickness of the intestinal wall was $>6.5 \mathrm{~mm}$ and the difference was statistically significant $\left(\chi^{2}=4.72, \mathrm{P}<0.05\right.$; Table III). Other findings included oedema and increased density of the mesentery $(n=15,65.2 \%)$; increased density of subcutaneous fat and blurred and swollen pelvic wall muscles $(n=4,17.4 \%)$, with the obturator internus and levator ani muscles being most commonly affected; narrowed intestinal lumen $(n=3,13.0 \%)$; and a small amount of ascitic fluid $(n=2,8.7 \%)$ located in the paracolic sulci and bladder or Douglas pouch. The thickness of the bladder wall was increased evenly $(n=5,21.7 \%)$; patients with urocystitis $(n=2)$ were diagnosed using a cystoscope (Fig. 2). One patient underwent $\mathrm{CT}$ and colonoscopic examination prior to and after radiotherapy (Fig. 3). Through comparing the findings, the CT manifestations and colonoscopic changes of CRC\&R were evaluated. A rectovaginal fistula was found in 1 patient and pelvic abscess was identified in 1 patient by $\mathrm{CT}$.

Colonoscopy. The 23 patients underwent colonoscopy and were diagnosed with RC\&R. The diagnoses included telangiectasia and mucosal hyperaemia $(n=21,91.3 \%)$, rectal ulcers $(n=3,13.0 \%)$ and narrowed intestinal lumen, which made it difficult for the colonoscope to pass through $(n=3,13.0 \%)$. A total of 2 patients were subjected to biopsy and chronic mucosal inflammation was confirmed.

\section{Discussion}

$\mathrm{RE}$ is a common complication of radiotherapy for pelvic malignant tumours and is reported in $5-15 \%$ of such cases (8). It is generally thought that the occurrence of RE is more likely when the pelvic external radiation dose exceeds 50 Gy delivered within 5 weeks (9).

According to the clinical onset time and symptoms, RE may be classified as ARE or CRE. The symptoms of ARE mostly occur within 1-2 weeks after radiotherapy and last for several weeks, and usually subside with conservative treatment. By contrast, CRE is a slow process and may occur 3 months or as long as 30 years after the completion of treatment. In addition 
A

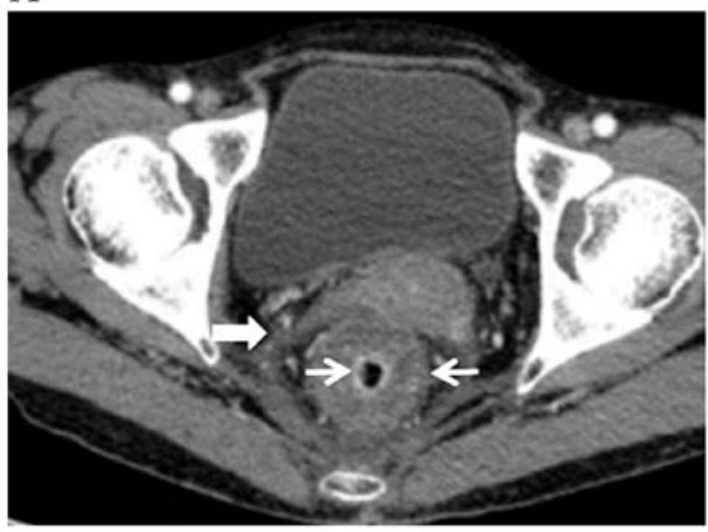

$\mathrm{C}$

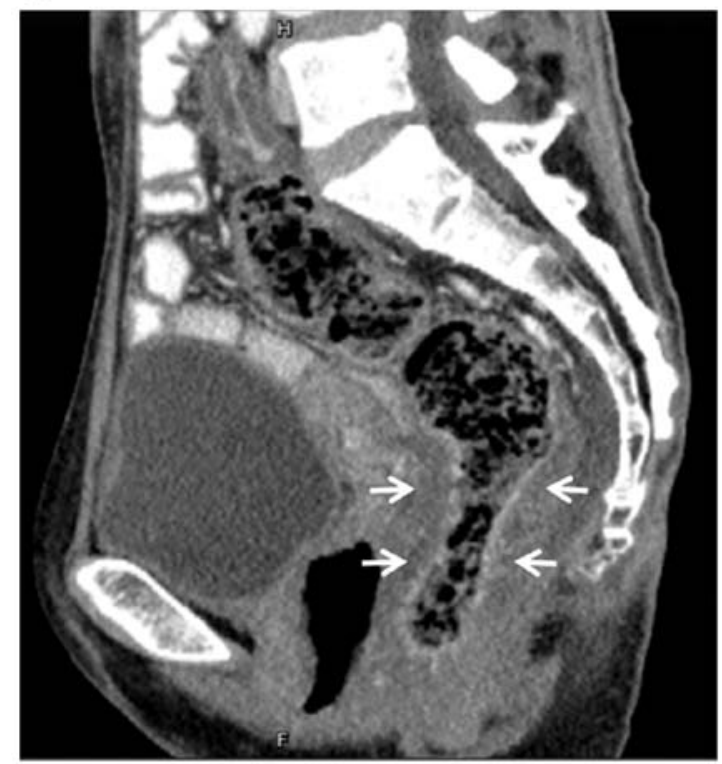

B

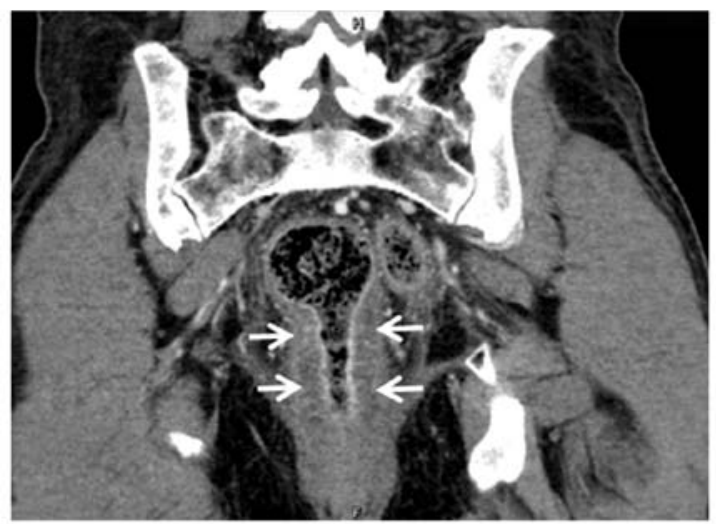

$\mathrm{D}$

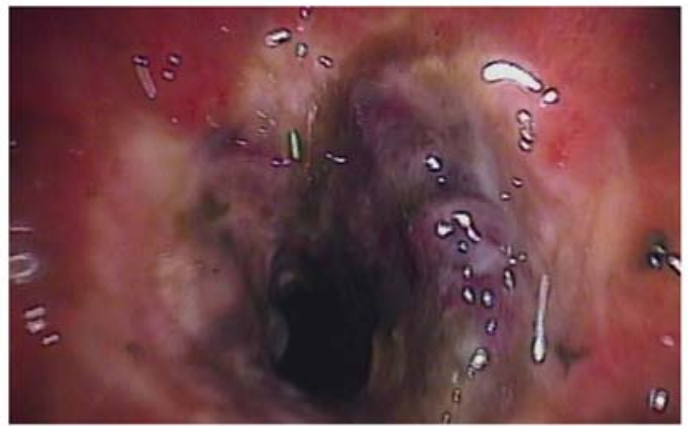

Figure 1. A 42-year-old female patient with cervical cancer and chronic radiation rectitis. The intestinal wall exhibited an evenly increased thickness to $16.6 \mathrm{~mm}$. (A) The 'target sign' ( $\mathrm{n}=16,69.9 \%)$ was observed on transverse sections as an obviously enhanced mucosa and/or serosa and appears as three rings of alternating attenuation (thin arrows) and thickened rectal fascia (bold arrowMultiplanar reconstruction (B) coronal and (C) sagittal scans demonstrated the lesions in more detail (thin arrows). (D) Colonoscopy revealed obvious oedema and hyperaemia of the rectal mucosa.

to acute cytotoxicity, radiation can cause progressive occlusive arteritis and submucosal fibrosis. Transmucosal damage to the intestinal wall may lead to progressive vasculitis, thrombosis, and eventually to varying degrees of ischemia and necrosis. This process may lead to narrowing of the intestinal lumen and eventual blockage. The chronic effects of radiation are mainly related to the total dose of radiation received and the total volume of the irradiated tissue (4).

CRC\&R is a common complication of radiotherapy for pelvic malignant tumours and is associated with a variable latency period. The time interval from the beginning of radiotherapy to diagnosis and CT and endoscopy examination in the present study varied between 4 and 28 months, and all patients exhibited late RE. Previously reported evidence suggests that chronic radiation proctitis is more likely to occur in those initially experiencing severe acute proctitis (10), which is referred to as 'the consequential late effect' (11). However, not all CRC\&R cases develop following an acute phase. Additional factors that may increase the incidence of CRC\&R were also identified. Mounting evidence has demonstrated that previous surgeries may increase the incidence rate of CRC\&R, which may be attributed to partly fixed intestinal loops by postoperative adhesions with excessive radiation exposure $(12,13)$. A correlation also exists between the selection of various surgical methods for different diseases and RE caused by postoperative adhesions (12). In addition, pelvic inflammatory disease, radiation dose, diabetes, cardiovascular diseases and smoking may serve as facilitators of CRC\&R (14).

Various symptoms and signs may occur in patients with CRC\&R, including chronic abdominal pain, constipation, ileus and haematochezia (15). In the present study, the majority of the patients suffered from abdominal pain $(n=15$, $52.2 \%$ ) with increased frequency of defecation and haematochezia $(n=16,69.6 \%)$. For any patient with a history of abdominal or pelvic irradiation, if gastrointestinal discomfort occurs, radiation damage should be considered, unless there is a diagnosis of other gastrointestinal diseases. For those patients with CRC\&R, preoperative imaging may help the surgeons plan the surgical strategy (16). At present, the radiological assessment of CRC\&R mostly involves barium enema. A series of studies have verified that the major radiological 
Table III. Association between intestinal wall thickness and 'target signs'.

\begin{tabular}{|c|c|c|c|c|}
\hline Intestinal wall thickness (mm) & 'Target sign' & No 'target sign' & Total & Statistics \\
\hline$\leq 6.5$ & 1 & 4 & 5 & $\chi^{2}=4.72(\mathrm{P}<0.05)$ \\
\hline$>6.5$ & 15 & 3 & 18 & \\
\hline Total & 16 & 7 & 23 & \\
\hline
\end{tabular}

A

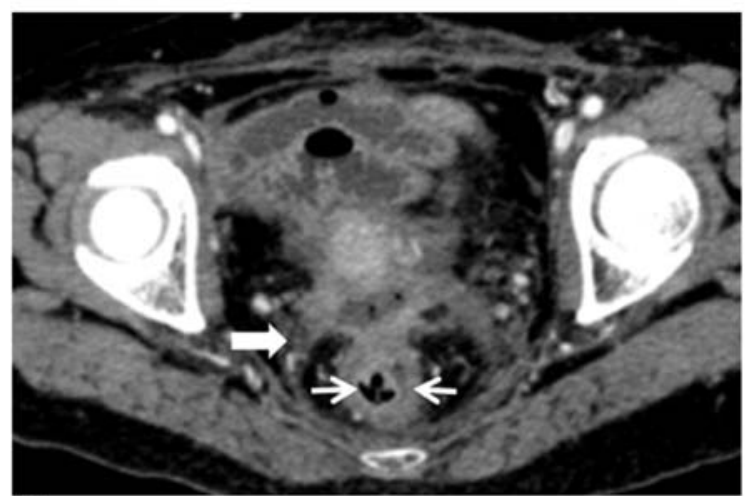

$\overline{\mathrm{C}}$

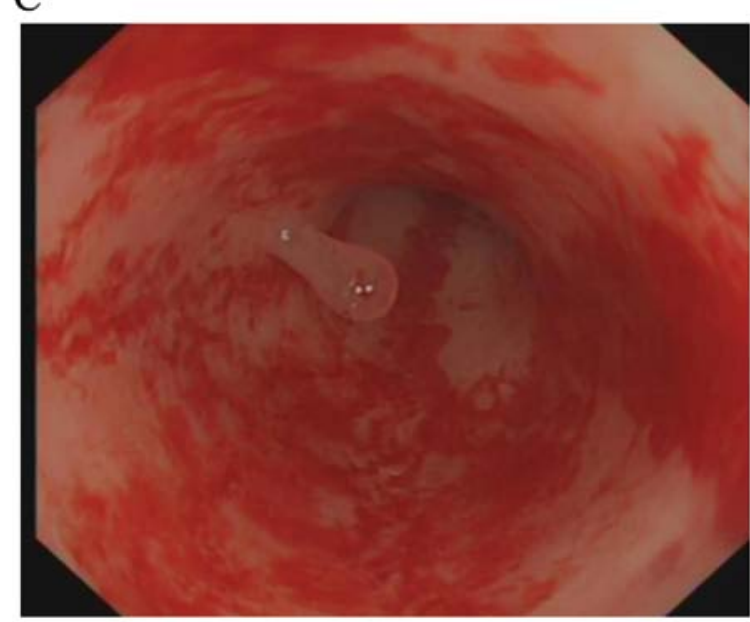

B

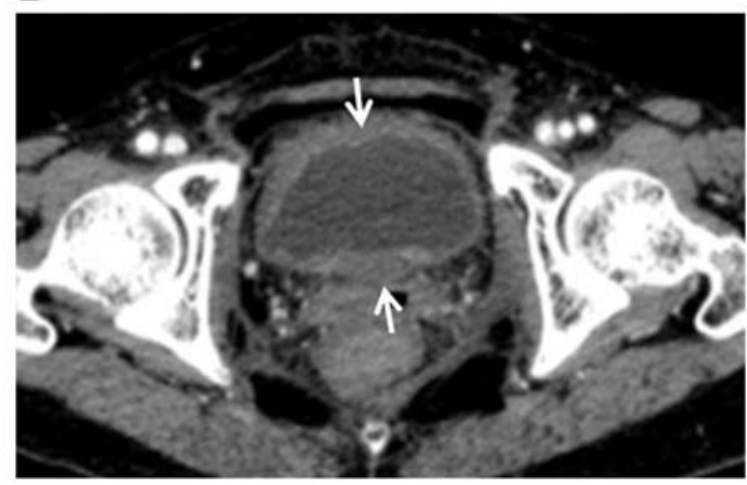

D

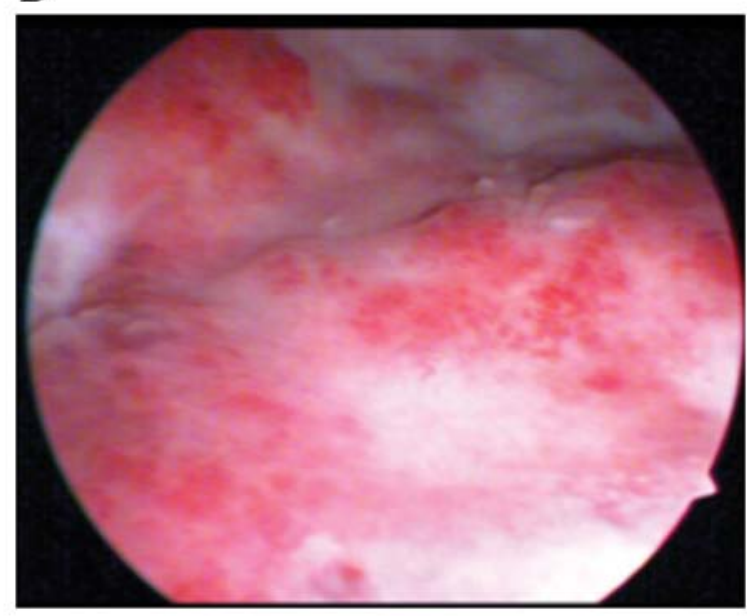

Figure 2. A 50-year-old female patient with cervical cancer and chronic radiation rectitis. The intestinal wall displayed an evenly increased thickness to $8.5 \mathrm{~mm}$. (A) The 'target sign' ( $\mathrm{n}=16,69.9 \%)$ was observed on transverse sections as an obviously enhanced mucosa and/or serosa, stratified changes (thin arrows) and thickened rectal fascia (bold arrow). (B) The bladder wall was thickened (thin arrow). (C) Colonoscopy revealed oedema and hyperaemia of the rectal mucosa with scattered malformed blood vessels. (D) On cystoscopy, oedema and hyperaemia of the bladder mucosa were observed.

findings varied from normal (15\% of the initial examinations) to decreased distensibility of the bowel wall, intestinal fixation, mucosal and contour abnormalities, ulceration, stenoses and fistula formation (17-19). The 'omega sign', caused by bilateral retraction at the base of a narrowed sigmoid loop was detected in $\sim 60 \%$ of patients with radiation colitis. In addition, due to the more accurate assessment of mucosal abnormalities and ulcers, colonoscopy is frequently applied in the evaluation of CRC\&R. The symptoms of CRC\&R include a thickened and hardened mucosa, as well as the expansion, ulceration, stenosis and necrosis of capillaries, among which expansion is the most common finding. Colonoscopy is able to readily identify intestinal mucosal lesions or may be used for biopsy and pathological evaluation in cases with suspected malignancy. Without colonoscopy and biopsy, it is difficult to distinguish CRC\&R from intestinal tumours or other inflammatory lesions. Due to the risk of fistula formation, the decision to perform a biopsy should be made with caution. If possible, the anterior rectal wall, which is usually exposed to high radiation doses, should be avoided (20). In the present study, following the diagnosis of RE via colonoscopy, the risk of fistulas was also considered; only 2 patients, in whom large ulcers were identified on colonoscopy, underwent biopsy to eliminate malignant tumours, and were ultimately diagnosed with chronic mucosal inflammation. As an invasive examination, in addition to not being suitable for high-risk patients, colonoscopy is also associated with pain and the risk of perforation; by contrast, $\mathrm{CT}$ is a non-invasive, safe and efficient method for almost all patients, including those considered as high-risk (21). 
A

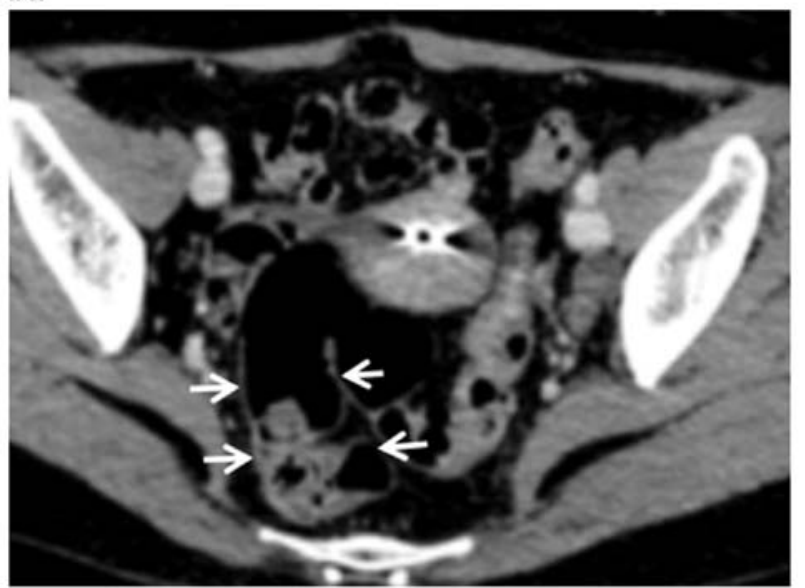

C

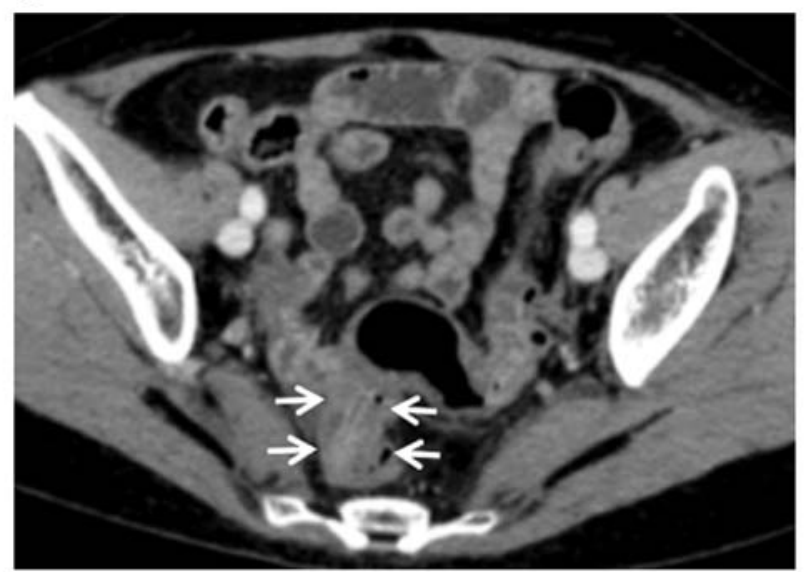

B

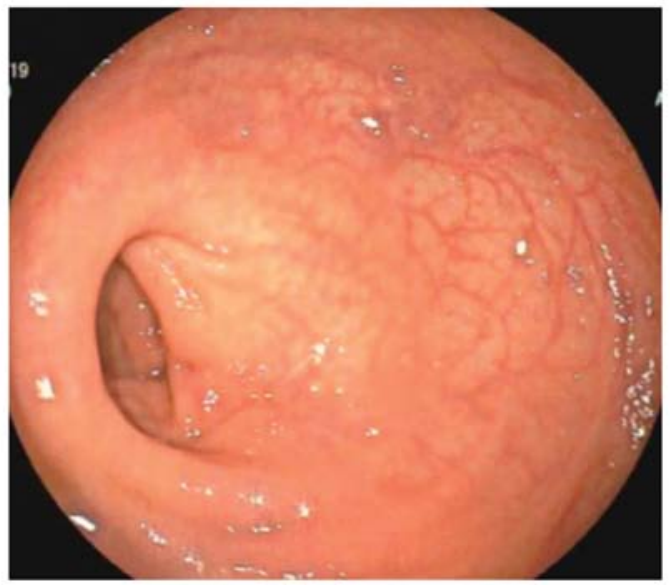

$\mathrm{D}$

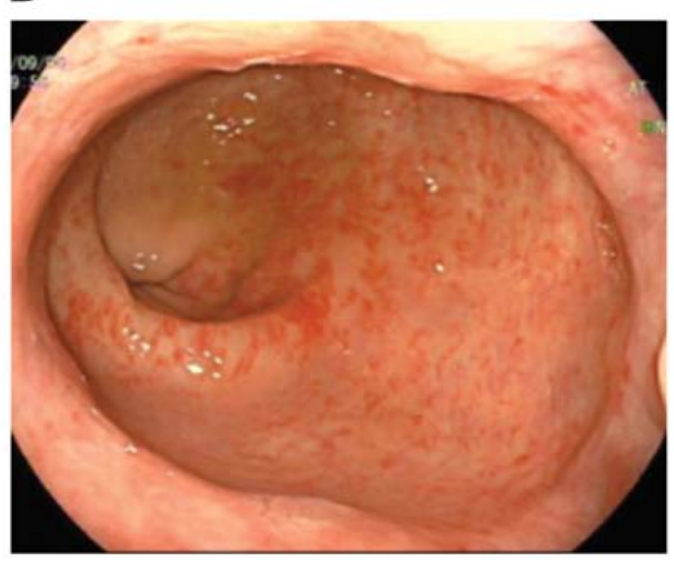

Figure 3. A 62-year-old female patient with cervical cancer. The findings on CT and colonoscopy prior to and after radiotherapy were compared. (A) Prior to radiotherapy, pelvic CT demonstrated no increase in the thickness of the intestinal wall (thin arrow). (B) Prior to radiotherapy, colonoscopy revealed no abnormalities. (C) At 1 year post-radiotherapy, the CT examination revealed an evenly increased thickness of the intestinal wall to 7.8 mm. (D) At 1 year post-radiotherapy, colonoscopy demonstrated oedema and hyperaemia of the mucosa of the sigmoid colon and rectum. The diagnosis of chronic radiation colitis and rectitis was considered.

At present, only few MSCT studies on CRC\&R are available $(21,22)$. In the present study, based on the abovementioned data, the MSCT characteristics of CRC\&R may be summarized as follows: i) The highest incidence (96.7\%) was in patients with rectal lesions, followed by $30.5 \%$ in those with sigmoid colon lesions and $26.1 \%$ in those with both rectal and sigmoid colon lesions. It has been demonstrated that the intestinal sensitivity to radiation varies among different sites, and the order from highest to lowest is as follows: Rectum, sigmoid colon, transverse colon, ileum, jejunum and duodenum, consistently with data reported by relevant studies $(23,24)$. ii) Intestinal changes included thickening of the involved intestinal walls and most cases exhibited a thickening of no more than $10 \mathrm{~mm}$, and even thickening of the smooth muscle layer and entire intestinal wall. When an obvious submucosal oedema occurred, the 'target sign' was observed on the enhanced scans (6), In the present study, the majority of patients (87\%) exhibited even thickening of the intestinal walls, among whom $69.6 \%$ displayed the target sign. In addition, when the thickness of the intestinal wall was $>6.5 \mathrm{~mm}$, the target sign was more commonly observed $(\mathrm{P}<0.05)$, but further confirmation in a larger sample is required. Ileal ulcers, mostly of the incomplete type, were occasionally observed, which was likely the result of the narrowed intestinal lumen caused by fibrosis and chronic inflammation-associated adhesions to the surrounding tissues. iii) Changes in the mesentery, peritoneum and pelvic wall: Following radiotherapy, widespread oedema occurred in the subcutaneous fat, mesentery and pelvic wall soft tissues, with interstitial and fibrous tissue proliferation in the late stages. On CT, an increased density but blurred appearance of the subcutaneous fat was observed, with common cords, mesh intervals, thickened mesenteric vessels, blurred edges, swollen pelvic wall muscles and blurred muscle intervals; the obturator internus and externus and levator ani muscles were the ones most commonly affected. The peritoneum and pelvic fascia were observed to be thickened, particularly the rectal fascia. iv) Abdominal cavity changes: When the intestinal wall ischemia progresses further, the ulcers may perforate the intestinal wall and cause peritonitis and formation of a pelvic abscess or an intestinal fistula. The pelvic abscess may be confined to the vicinity of the lesions and the low-lying area of the pelvic cavity away from the crevasse. The abscess borders may be better displayed on enhanced CT scans. Sinus tracts may be frequently difficult to image directly using CT, but their presence may be indicated by luminal fluid and 
gases. Due to the weakened tissue repair capacity caused by radiotherapy and constant leakage of intestinal contents, it is difficult for the sinus tract to close spontaneously and surgical treatment is frequently required (25). A small amount of ascitic fluid is rarely observed. v) Other changes: Radiation may also damage other organs in the pelvic cavity, particularly the urinary system, manifesting as radiation-induced urocystitis and inflammatory stenosis of the lower ureter. In the present study, $21.7 \%$ of patients had a thickened bladder wall and 2 patients had confirmed radiation-induced cystitis. In addition, MSCT has a variety of image post-processing modes, and the multiplanar reconstruction (MPR) is able to display the characteristics of thickened intestinal walls and surrounding tissues, allowing for a comprehensive evaluation of the intestinal walls.

The appearance of CRE on imaging lacks specificity and it must be clinically distinguished from intestinal tumours, inflammatory bowel disease and ischemic bowel disease. Intestinal tumours frequently invade the intestinal wall and form a mass, which may be easily distinguished from the intestinal wall thickening observed in CRE. Inflammatory lesions are more common in ulcerative colitis and Crohn's disease, both of which have characteristic predominantly affected sites. Crohn's disease is characterized by multiple segment involvement. Both may display irregular thickening of the intestinal wall, stenosis, intestinal ulcers, even formation of fistulas or pelvic abscesses. However, the changes in the mesentery and pelvic wall are minor and consideration of the patient's history may help distinguish it from CRE (26). Acute ischemic bowel disease frequently manifests as oedema and thickening of the intestinal wall, oedema and blurred appearance of the mesentery, and it is difficult to distinguish from CRE. Ischemic bowel disease is mostly caused by vasculitis, trauma, intestinal obstruction, intestinal torsion or vascular sclerosis. There is no evidence suggesting that the development of chemic bowel disease is related to radiotherapy. Intestinal wall gas is a characteristic manifestation of intestinal ischemic necrosis (27).

In summary, the CT manifestations of CRC\&R are variable and non-specific but are easily diagnosed when combined with a history of radiotherapy and clinical manifestations. The MSCT of CRC\&R exhibits certain distinct characteristics, including an evenly thickened intestinal wall, particularly in the rectum, mostly increase to $<10 \mathrm{~mm}$. When submucosal oedema develops, particularly in cases with wall thickening to $>6.5 \mathrm{~mm}$, the 'target sign' may be observed through enhanced CT scans. An increased density but blurred appearance of the subcutaneous fat may be observed, with common cords, mesh intervals, thickened mesenteric vessels, blurred edges, swollen pelvic wall muscles and blurred muscle borders. In addition to demonstrating the intestinal lesions, CT also reveals changes outside the intestine, particularly mesenteric oedema and pelvic abscesses, which may prompt early clinical treatment measures. MPR is able to display the characteristics of a thickened intestinal wall and surrounding tissues, allowing for a comprehensive evaluation of the intestinal walls.

\section{Acknowledgements}

Not applicable.

\section{Funding}

No funding was received.

\section{Availability of data and materials}

The datasets used and/or analyzed during the current study are available from the corresponding author on reasonable request.

\section{Authors' contributions}

JX and QDL designed the experiments, analyzed the data and wrote the manuscript. Both authors read and approved the final manuscript.

\section{Ethics approval and consent to participate}

The present study was approved by the Ethics Committee of the Chongqing University Cancer Hospital (approval no. 2020060) and written informed consent was obtained from all participants.

\section{Patient consent for publication}

Not applicable.

\section{Competing interests}

The authors declare that they have no competing interests.

\section{References}

1. Kennedy GD and Heise CP: Radiation colitis and proctitis. Clin Colon Rectal Surg 20: 64-72, 2007.

2. Shadad AK, Sullivan FJ, Martin JD and Egan LJ: Gastrointestinal radiation injury: Prevention and treatment. World J Gastroenterol 19: 199-208, 2013.

3. Li YS: Radiation pelvicopathy: A comprehensive and interdisciplinary approach. J Med Postgrad 29: 449-452, 2016.

4. Regimbeau JM, Panis Y, Gouzi JL and Fagniez PL; French University Association for Surgical Research: Operative and long term results after surgery for chronic radiation enteritis. Am J Surg 182: 237-242, 2001.

5. Zhang XH, Gao MY, Zhou XH, et al: MRI diagnosis of radiation enteritis in patients with gynecological pelvic malignancies after radiotherapy. Chin J Med Imaging Technol 28: 1695-1698, 2012.

6. Chen S, Harisinghani MG and Wittenberg J: Small bowel CT fat density target sign in chronic radiation enteritis. Australas Radiol 47: 450-452, 2003.

7. Harb AH, Abou Fadel C and Sharara AI: Radiation enteritis. Curr Gastroenterol Rep 16: 383, 2014.

8. Zimmerer T, Böcker U, Wenz F and Singer MV: Medical prevention and treatment of acute and chronic radiation induced enteritis-is there any proven therapy? A short review. Z Gastroenterol 46: 441-448, 2008.

9. DeVita VT, Hellman S and Rosenberg SA (eds): Cancer: Rrinciples and practice of oncology. Philadelphia, New York, Lippincott Williams and Wilkins Press, pp1527-1528, 2008.

10. Denham JW, O'Brien PC, Dunstan RH, Johansen J, See A, Hamilton CS, Bydder S and Wright S: Is there more than one late radiation proctitis syndrome? Radiother Oncol 51: 43-53, 1999.

11. Dorr W and Hendry JH: Consequential late effects in normal tissues. Radiother Oncol 61: 223-231, 2001.

12. Huang Y, Guo F, Yao D, Li Y and Li J: Surgery for chronic radiation enteritis: Outcome and risk factors. J Surg Res 204: 335-343, 2016.

13. Theis VS, Sripadam R, Ramani V and Lal S: Chronic radiation enteritis. Clin Oncol (R Coll Radiol) 22: 70-83, 2010. 
14. Iraha S, Ogawa K, Moromizato H, Shiraishi M, Nagai Y, Samura H, Toita T, Kakinohana Y, Adachi G, Tamaki W, et al: Radiation enterocolitis requiring surgery in patients with gynecological malignancies. Int J Radiat Oncol Biol Phys 68: 1088-1093, 2007.

15. Krol R, Smeenk RJ, van Lin EN and Hopman WP: Impact of late anorectal dysfunction on quality of life after pelvic radiotherapy. Int J Colorectal Dis 28: 519-526, 2013.

16. Haddad MC, Khouzami RA,Saad HA and Azzi MC: Imaging fifindings of radiation enteritis. J Med Liban 52: 55-57, 2004.

17. Smith DH and DeCosse JJ: Reliation damage to the small intestine. World J Surg 10: 189-194, 1986.

18. Chater C, Saudemont A and Zerbib P: Chronic radiation enteritis. J Visc Surg 156: 175-176, 2019.

19. Zhang SY and LI YS: Diagnosis of chronic radiation enteritis J Med Postgrad 25: 654-657, 2012.

20. Wu XR, Liu XL, Katz S and Shen B: Pathogenesis, diagnosis, and management of ulcerative proctitis, chronic radiation proctopathy, and diversion proctitis. Inflamm Bowel Dis 21: 703-715, 2015.

21. Raman SP, Horton KM and Fishman EK: MDCT and CT angiography evaluation of rectal bleeding: The role of volume visualization. AJR Am J Roentgenol 201: 589-597, 2013.

22. Chen K, Yin F and Chen HT: Evaluation of clinical use of CT virtual colonoscopy. China Prac Med 4: 115-116, 2009.
23. Kalaoselvan R, Theis VS, Dibb M, Teubner A, Anderson ID, Shaffer JL, Carlson GL and Lal S: Radiation enteritis leading to intestinal failure: 1994 patient-years of experience in a national referral centre. Eur J Clin Nutr 68: 166-170, 2014.

24. Kasuya G, Ogawa K, Iraha S, Nagai Y, Shiraishi M, Hirakawa M, Samura H, Toita T, Kakinohana Y, Kudaka W, et al: Severe late complications in patients with uterine cancer treated with postoperative radiotherapy. Anticancer Res 31: 3527-3533, 2011.

25. Chen MC, Chiang FF, Hsu TW, Chen JB, Chao TH, Ma HF and Wang HM: Clinical experience in 89 consecutive cases of chronic radiation enterocolitis. J Chin Med Assoc 74: 69-74, 2011.

26. Seastedt KP, Trencheva K, Michelassi F, Alsaleh D, Milsom JW, Sonoda T, Lee SW and Nandakumar G: Accuracy of CT enterography and magnetic resonance enterography imaging to detect lesions preoperatively in patients undergoing surgery for Crohn's disease. Dis Colon Rectum 57: 1364-1370, 2014.

27. Cox VL, Tahvildari AM, Johnson B, Wei W and Jeffrey RB: Bowel obstruction complicated by ischemia: Analysis of CT findings. Abdom Radiol (NY) 43: 3227-3232, 2018.

This work is licensed under a Creative Commons Attribution-NonCommercial-NoDerivatives 4.0 International (CC BY-NC-ND 4.0) License. 